\title{
ANTIOXIDANTS ATTENUATE HYPERGLYCAEMIA-MEDIATED BRAIN \\ ENDOTHELIAL CELL DYSFUNCTION AND BLOOD-BRAIN BARRIER HYPERPERMEABILITY
}

Claire L. Allen, Ulvi Bayraktutan

Division of Stroke Medicine, Clinical Sciences Building, University of Nottingham, UK.

Address for correspondence

Ulvi BAYRAKTUTAN BVMS, DVM, PhD

Associate Professor,

Division of Stroke Medicine,

Clinical Sciences Building,

University of Nottingham,

Hucknall Road,

Nottingham NG5 1PB

United Kingdom

Tel.: +44 (115)8231764

Fax: +44 (115)8231767

E-mail: ulvi.bayraktutan@nottingham.ac.uk 


\begin{abstract}
Aims: Hyperglycaemia, in stroke patients, is associated with worse neurological outcome by comprimising endothelial cell function and the blood-brain barrier (BBB) integrity. We have studied the contribution of hyperglycaemia-mediated generation of oxidative stress to these pathologies and examined whether antioxidants as well as normalisation of glucose levels following hyperglycaemic insult reverse these phenomena.
\end{abstract}

Methods: Human brain microvascular endothelial cell (HBMEC) and human astrocyte cocultures were used to simulate the human BBB. The integrity of the BBB was measured by transendothelial electrical resistance (TEER) using STX electrodes and an EVOM resistance meter while enzyme activities were measured by specific spectrophotometric assays.

Results: After 5 days of hyperglycaemic insult there was a significant increase in BBB permeability which was reversed by glucose normalisation. Co-treatment of cells with hyperglycaemia and a number of antioxidants including vitamin $\mathrm{C}$, free radical scavengers and antioxidant enzymes including catalase and superoxide dismutase mimetics attenuated the detrimental effects of hyperglycaemia. Inhibition of p38 MAPK and protein kinase C but not PI3 kinase also reversed hyperglycaemia-induced BBB hyperpermeability. In HBMEC, hyperglycaemia enhanced pro-oxidant $(\mathrm{NAD}(\mathrm{P}) \mathrm{H}$ oxidase) enzyme activity and expression which were normalised by reverting to normoglycaemia.

Conclusions: Hyperglycaemia impairs brain microvascular endothelial function through involvements of oxidative stress and several signal transduction pathways. 


\section{Introduction}

Vascular endothelium covers the entire inner surface of all the blood vessels and is implicated in the regulation of vasomotor tone, cellular trafficking and the local balance between pro- and anti-inflammatory mediators $[1,2]$. Endothelial cells in some organs differentiate and develop into specialised barriers to regulate vascular permeability. One such example is the blood-brain barrier (BBB) which stops the passage of substances from blood into the central nervous system. The BBB consists of brain microvascular endothelial cells (BMEC), capillary basement membranes and astrocyte end-feet. Astrocytes surround 99\% of the $\mathrm{BBB}$ endothelia and release soluble factors to maintain the integrity of the BBB [3]. Since the brain oedema, associated with the BBB disruption, is more prevalent in stroke patients with diabetes mellitus than those without, it is safe to suggest that hyperglycaemia plays a prominent role in this defect [4-6].

Diabetes mellitus is a metabolic disorder associated with microvascular complications. Although several mechanisms including non-enzymatic glycation of proteins and lipids with the irreversible formation/deposition of advanced glycation end products (AGEs), the activation of polyol pathway, stimulation of protein kinase $\mathrm{C}(\mathrm{PKC})$ or oxidative stress accompanied by overproduction of reactive oxygen species (ROS) have been implicated in peripheral vascular pathologies [1], the mechanisms by which hyperglycaemia exerts damage on brain microvasculature remain unknown.

The hyperglycaemia-mediated activation of PKC represents a hallmark in peripheral vasculopathies and is associated with increases in endothelial permeability, angiogenesis and ROS overproduction $[7,8]$. ROS, in particular superoxide anion $\left(\mathrm{O}_{2}^{\left.-{ }^{-}\right)}\right.$increase vascular permeability in various disease models including focal ischaemia and impairs neuronal homeostasis and cerebrovascular tone $[9,10]$. NAD(P)H oxidase, a pro-oxidant enzyme with a membrane-bound active core consisting of p22-phox and gp91-phox subunits, has been 
shown as the main enzymatic source of ROS in coronary EC exposed to hyperglycaemia [11]. Under physiological conditions, once generated $\mathrm{O}_{2}{ }^{--}$is neutralised by superoxide dismutases (SODs) to $\mathrm{H}_{2} \mathrm{O}_{2}$ which in turn is metabolised to $\mathrm{H}_{2} \mathrm{O}$ by catalase and glutathione peroxidase (GPx).

Nitric oxide (NO), another ROS generated by endothelial NO synthase (eNOS), is also implicated in diabetic microvasculopathy and BBB hyperpermeability in cerebral pathologies such as middle cerebral artery occlusion where the NOS inhibitors (L-NNA and L-NAME) have been shown to reduce both brain oedema and BBB damage [12]. However, physiological levels of NO are known to regulate cerebral blood flow, vascular tone and protect against ischaemic stroke by increasing collateral flow to the ischaemic area [13]. It is of note that endothelial cells represent the main cellular source of $\mathrm{NO}$ and $\mathrm{O}_{2}{ }^{--}$and the prevalence of diabetes is significantly greater in ischaemic stroke patients with small-vessel compared to large-artery diseases [14].

It is well-documented that hyperglycaemia promotes a pro-coagulant state to compromise blood supply to the penumbral areas in acute ischaemic stroke (AIS) through, in part, increasing the expressions of intercellular adhesion molecule-1 (ICAM-1) and vascular cell adhesion molecule-1 (VCAM-1) [15].

In the light of the currently available data, this study hypothesises that hyperglycaemia may perturb endothelial function and the BBB integrity by promoting oxidative stress and employing a variety of intermediary molecules. To this end, the present study was designed to investigate whether; i- changes in the expression and/or activities of oxidative stress related parameters i.e. adhesion molecules and pro- and antioxidant enzymes are involved in hyperglycaemia-mediated cerebral microvascular endothelial cell dysfunction, ii- exposure of HBMEC and human astrocytes (HA) co-cultures to normoglycaemia or hyperglycaemia distinctly modulate blood-brain barrier (BBB) integrity; iii- antioxidants and signal 
transduction pathway inhibitors can help in the maintenance of the BBB integrity during hyperglycaemic insult; iv- normalisation of glucose levels has favourable effects on the aforementioned parameters following hyperglycaemic insult.

\section{Materials and Methods}

\section{Cell culture}

HBMEC and HA were purchased from TCS Cellworks (Buckingham, UK) and cultured in specialised media. HA were characterised by glial fibrillary acidic protein (GFAP) staining while HBMEC were characterised by stainings for vWF/Factor VIII and CD31 (P-CAM) as well as by uptake of DiI-Ac-LDL. Cells were grown to subconfluence and incubated under normoglycaemic (NG; D-Glucose; $5.5 \mathrm{mM}$ ) and hyperglycaemic (HG; D-Glucose; $25 \mathrm{mM}$ ) conditions for a period of five days. Cells were also cultured in equimolar concentrations of D-Mannitol (25 $\mathrm{mM})$ to assess if the changes observed in enzyme activities or expressions were due to hyperosmolality rather than HG per se. In a sub-set of experiments HBMEC were

initially cultured in hyperglycaemic conditions for 5 days followed by a further 5 days culture in normoglycaemic conditions to assess the specific effects of glucose normalisation on aforementioned oxidative stress-related biomarkers.

\section{Evaluation of cell viability}

To detect cytotoxicity of outlined treatment regimens, a small aliquot of cells were incubated in $0.1 \%$ trypan blue for 4 min and viewed under a light microscope. By counting 100 cells, the percentage of viable cells was calculated.

\section{In vitro BBB co-culture model}

HA were seeded on the outside of collagen-coated polycarbonate membrane $(0.4 \mu \mathrm{M})$ Transwell inserts (12-well type, Corning) directed upside down in the culture chamber. HA were allowed to adhere to the membrane overnight and then inverted the correct way. HBMEC were seeded on the inside of the insert and cells were grown to confluence, to create 
the contact co-culture model. The non-contact model is a replica of the contact model but the astrocytes were seeded onto the bottom of the well as opposed to the underside of the Transwell insert. Co-cultures were incubated for a period of $72 \mathrm{~h}$ under NG, HG and HG in the presence of an antioxidant agent including vitamin $\mathrm{C}(0.5 \mu \mathrm{M})$, catalase $(150 \mathrm{U} / \mathrm{mL})$, a free radical scavenger (MnTBAP; $50 \mu \mathrm{M}$ ), a protein kinase $\mathrm{C}$ inhibitor i.e. bisindolyamide I $(5 \mu \mathrm{M})$ or a MAPK inhibitor (SB203580; $10 \mu \mathrm{M})$. To investigate the extent of HA involvement in preservation of the BBB integrity under $\mathrm{HG}$ conditions, a monolayer of HBMEC were exposed to the aforementioned agents.

\section{Measurement of BBB integrity}

This was assessed by measuring transendothelial electrical resistance (TEER) across the membrane using STX electrodes and an EVOM resistance meter (World Precision Instruments, UK). Three independent readings were taken every 24 hours and blank filter measurements were subtracted from inserts seeded with cells. Values are shown as $\Omega \mathrm{cm}^{2}$ based on culture insert size.

\section{Measurement of NAD(P)H oxidase activity}

$\mathrm{NAD}(\mathrm{P}) \mathrm{H}$ oxidase activity was measured by cytochrome $C$ reduction assay. Briefly, an aliquot of HBMEC homogenates (obtained from $20 \times 10^{6}$ cells by homogenisation of the cell pellet in cold 20 mM HEPES buffer, pH 7.2, containing 1 mM EGTA, 210 mM mannitol and $70 \mathrm{mM}$ sucrose) was incubated with $50 \mu \mathrm{M}$ cytochrome $C$ for $60 \mathrm{~min}$ at $37^{\circ} \mathrm{C}$. $\mathrm{O}_{2}^{-{ }^{-}}$generation was measured as the superoxide dismutase $(10 \mu \mathrm{g} / \mathrm{ml})$-inhibitable reduction of cytochrome $C$ and monitored as the change in absorbance at $550 \mathrm{~nm}$ using a GENios plate reader (TECAN). In order to ascribe all cytochrome $\mathrm{C}$ reduction to $\mathrm{NAD}(\mathrm{P}) \mathrm{H}$ oxidase, the specific inhibitors of other ROS-generating enzymes i.e. L-NAME (100 $\mu \mathrm{M}$, NOS inhibitor), rotenone (50 $\mu \mathrm{M}$, mitochondrial complex I inhibitor), allopurinol (100 $\mu \mathrm{M}$, xanthine oxidase inhibitor) or indomethacin (50 $\mu \mathrm{M}$, cyclooxygenase inhibitor) were added simultaneously to aliquots at the 
beginning of 60 min incubation period prior to determining $\mathrm{O}_{2}{ }^{--}$generation. Absorbances were recorded for $8 \mathrm{~min}$ with $60 \mathrm{sec}$ intervals and activity was calculated as pmoles $\mathrm{O}_{2}{ }^{-{ }^{-}}$per $\mathrm{mg}$ protein following subtraction of background levels at $550 \mathrm{~nm}$.

\section{NOS Assay}

NOS activity was determined, in cell homogenates, using the NOSdetect assay kit (Alexis Biochemicals). Briefly, cells were lysed in buffer containing $25 \mathrm{mM}$ Tris, $\mathrm{pH} 7.4,1 \mathrm{mM}$ EDTA and $1 \mathrm{mM}$ EGTA and centrifuged for $30 \mathrm{~min}$ at $4000 \mathrm{~g} .10 \mu \mathrm{l}$ supernatant and $40 \mu \mathrm{L}$ reaction buffer (50 mM Tris, $\mathrm{pH}$ 7.4, $6 \mu \mathrm{M}$ tetrahydrobiopeterin, $2 \mu \mathrm{M}$ flavin adenine mononucleotide, $2 \mu \mathrm{M}$ flavin adenine dinucleotide, $10 \mathrm{mM} \mathrm{NADPH}, 6 \mathrm{mM} \mathrm{CaCl} 2$ ) containing $100.000 \mathrm{dpm}\left[{ }^{3} \mathrm{H}\right] \mathrm{L}$-arginine were combined. Following $30 \mathrm{~min}$ incubation at room temperature, the enzymatic reaction was stopped by the addition of stop buffer $(50 \mathrm{mM}$ HEPES, 5 mM EDTA, pH 5.5). Newly formed $\left[{ }^{3} \mathrm{H}\right] \mathrm{L}$-citrulline, neutral at $\mathrm{pH}$ 5.5, was then separated from the incubation mixture by cation exchange resin (Dowex AG $50 \mathrm{~W}-\mathrm{X} 8$ ) and quantified using a liquid scintillation counter. The contribution of iNOS to overall NOS activity was assessed in similar experiments by replacing the calcium in reaction buffer with EGTA $(5 \mathrm{mM})$. Data were normalised by the amount of protein and reaction time. Results were expressed as pmol L-citrulline/mg protein/min.

\section{Nitrite Detection}

Nitrite levels were measured by Griess reaction as an index of NO generation following conversion of nitrate to nitrite by nitrate dehydrogenase. Cellular homogenate was mixed with an equal volume of Griess reagent (sulfanilamide $1 \% \mathrm{w} / \mathrm{v}$, naphthylethylenediamine dihydrochloride $0.1 \% \mathrm{w} / \mathrm{v}$ and orthophosphoric acid $2.5 \% \mathrm{v} / \mathrm{v}$ ) and incubated at room temperature for $10 \mathrm{~min}$ prior to measurement of absorbances at $540 \mathrm{~nm}$. Nitrite formed was compared to those of known concentrations of sodium nitrite and normalised according to the protein concentration. 


\section{SOD Assay}

SOD activity was measured by detecting the level of variation in $\mathrm{O}_{2}{ }^{--}$levels using a tetrazolium salt. Untreated and treated HBMEC homogenates were diluted 1:20 with radical detector and assayed in triplicate in a 96-well ELISA plate (Calbiochem). Reactions were initiated by the addition of $20 \mu \mathrm{l}$ diluted xanthine oxidase to generate $\mathrm{O}_{2}{ }^{--}$and the plate was incubated on a shaker for $20 \mathrm{~min}$ at room temperature. Absorbances were read at $450 \mathrm{~nm}$ and total SOD activity was determined using the equation obtained from the linear regression of the SOD standard curve. One unit of SOD was defined as the amount of enzyme needed to exhibit 50\% dismutation of the $\mathrm{O}_{2}^{--}$(kit range 0.025-0.25 units/ml). MnSOD activity was detected following inhibition of CuZnSOD activity via incubation with $3 \mathrm{mM}$ potassium cyanide (BDH Chemicals Ltd, UK), at room temperature for 45 minutes. CuZnSOD activity was subsequently calculated by the subtraction of MnSOD activity from total SOD activities.

\section{Catalase Assay}

Catalase activity was measured using an immunoassay kit (Merck Biosciences, UK). The assay was based on the reaction of catalase with optimal concentration of $\mathrm{H}_{2} \mathrm{O}_{2}$ in the presence of methanol to produce formaldehyde and detect it spectrophotometrically using 4amino-3-hydrazino-5-mercapto-1,2,4-triazole (Purpald) as the chromogen. Enzyme activities were then determined using a standard curve generated with formaldehyde. One unit was defined as the amount of enzyme that generates the formation of $1 \mathrm{nmol}$ formaldehyde per $\min$, at $25^{\circ} \mathrm{C}$.

\section{GPx Assay}

GPx activity was measured using a specific immunoassay kit (Merck Biosciences, UK) based on the simultaneous reduction of glutathione and oxidation of $\mathrm{NAD}(\mathrm{P}) \mathrm{H}$ to $\mathrm{NADP}^{+}$, accompanied by a decrease in absorbance at $340 \mathrm{~nm}$. GPx activities were then calculated 
using the extinction coefficient for $\mathrm{NAD}(\mathrm{P}) \mathrm{H}$ at $340 \mathrm{~nm}\left(0.00373 \mu \mathrm{M}^{-1}\right)$. One unit of GPx was defined as the activity that converts $1 \mathrm{mM}$ of reduced glutathione per litre per min, at $25^{\circ} \mathrm{C}$.

\section{Cellular Adhesion Molecule Assays}

ICAM-1 and VCAM-1 concentrations were determined using quantitative sandwich enzyme immunoassay kits (R\&D Systems, UK) and microplates pre-coated with monoclonal antibodies specific for the markers in question, as per manufacturer's instructions. Sample absorbances were read at $450 \mathrm{~nm}$ and subtracted from readings taken at $540 \mathrm{~nm}$ or $620 \mathrm{~nm}$ for VCAM-1 and ICAM-1, respectively. Concentrations were then calculated from a standard curve of known values.

\section{Western Blotting}

Total cellular proteins were isolated by lysis and equal amounts of protein $(50 \mu \mathrm{g})$ were run on $10 \%$ SDS-polyacrylamide gels. Proteins were transferred to nitrocellulose membranes prior to overnight incubation with monoclonal primary antibodies. Primary antibodies to catalase, MnSOD, CuZnSOD and GPx were obtained from Merck Biosciences. eNOS was from Transduction Laboratories while those for gp91-phox and p22-phox were from Autogen Bioclear. Horseradish peroxidase linked secondary antibodies were then used and the bands were detected by enhanced chemiluminescence (GE Healthcare). Blots were assessed by densitometry of bands with subtraction of the background counts measured outside loaded lanes.

\section{Statistical Analysis}

Results of molecular assays and TEER experiments are presented as mean \pm SEM.

Statistical analyses were performed by two-way analysis of variance (ANOVA) followed by Bonferroni-Dunn's post hoc analysis and $\mathrm{p}<0.05$ was considered significant. 


\section{Results}

\section{Effects of hyperglycaemia on protein expression}

HG produced significant increases in p22-phox, a subunit of NAD $(\mathrm{P}) \mathrm{H}$ oxidase, eNOS, CuZnSOD, catalase and GPx protein expression in HBMEC without affecting that of MnSOD. Glucose normalisation following HG significantly decreased p22-phox, eNOS, catalase, and GPx protein levels (Figure 1).

\section{Effects of hyperglycaemia on pro-oxidant and antioxidant enzyme activities}

Exposure of HBMEC to HG for 5 days resulted in a 2-fold increase in pro-oxidant $\mathrm{NAD}(\mathrm{P}) \mathrm{H}$ oxidase enzyme activity compared to cells grown under normoglycaemic conditions. Normalisation of glucose levels for a further 5-day period attenuated the effects of the hyperglycaemic insult (Table 1).

Similar to NAD $(\mathrm{P}) \mathrm{H}$ oxidase, the eNOS activity and nitrite levels, a breakdown product of NO production, also rose in HBMEC cultured under hyperglycaemic conditions which were subsequently reduced by glucose normalisation (Table 1).

HG significantly enhanced CuZnSOD activity in HBMEC that was diminished by glucose normalisation where MnSOD activity appeared to be unaffected by HG (Table 1).

Catalase activity was found to be significantly increased in hyperglycaemic HBMEC that was diminished with glucose normalisation. Although hyperglycaemia produced a slight increase in GPx activities, glucose normalisation caused a profound decrease in its activity (Table 1).

\section{Effects of hyperglycaemia on adhesion molecules}

HG produced a significant increase in VCAM-1 but not ICAM-1 concentration. Glucose normalisation had no effect on ICAM-1 levels while significantly decreasing that of VCAM-1 compared to the HG group (Figure 2). 


\section{Effects of different treatment regimens on cellular viability}

No significant variations were observed in overall viability rates of HBMEC and HA cultured with HG, NG or hyperosmolar media (Table 2).

\section{Effects of hyperglycaemia on TEER}

As HA-HBMEC co-cultures produced better electrical resistance compared to non-contact and HBMEC monolayer models, this model has been used throughout the study to obtain statistically significant differences (Figure 3). HG induced a marked decrease in TEER compared to D-mannitol and NG treatment groups thereby inferring an increase in BBB permeability via disruption of the BBB where reversal of glucose to normal levels for $72 \mathrm{~h}$ restored BBB integrity (Figure 4). Treatment of cells with a wide range of antioxidants, namely an $\mathrm{NAD}(\mathrm{P}) \mathrm{H}$ oxidase inhibitor (apocynin; $500 \mu \mathrm{M})$, vitamin $\mathrm{C}(0.5 \mu \mathrm{M})$, a free radical scavenger (mercaptopropionylglycine (MPG); $0.5 \mu \mathrm{M}$ ), an antioxidant enzyme (catalase; $25-150 \mathrm{U} / \mathrm{ml}$ ) and two different SOD mimetics (MnTBAP; $50 \mu \mathrm{M}$ or Mn(III)TMPyP; $10 \mu \mathrm{M}$ ) restored HG-mediated decreases in TEER (Figure 5).

Other studies employing specific inhibitors of protein kinase C (Bisindolyamide I, $5 \mu \mathrm{M}$ ) and p38MAPK (SB203580, $10 \mu \mathrm{M})$ and PI3 kinase (LY29402, $50 \mu \mathrm{M})$ have shown that selective inhibitions of protein kinase $\mathrm{C}$ and p38MAPK but not PI3 kinase reverses HGinduced decreases in TEER readings (Figure 6).

\section{Discussion}

The major conclusions to be drawn from this study are that hyperglycaemia compromises the BBB integrity through mechanisms mediated, in part, by oxidative stress and different signal transduction pathways. Indeed, specific inhibition of PKC or p38MAPK pathways, alleviation of oxidative stress by a series of functionally distinct antioxidants and normalisation of high glucose levels restore the BBB integrity. 
Diabetes mellitus (DM) is a major risk factor for ischaemic stroke while hyperglycaemia without pre-existing diabetes is linked to a worse neurological outcome and stroke mortality $[9,16]$. Brain oedema constitutes one of the main causes of death following stroke and is associated with the $\mathrm{BBB}$ disruption. Considering that the brain oedema is more prevalent in stroke patients with DM than those without, it is reasonable to suggest that HG plays a prominent role in this defect [4-6]. Ischaemic stroke develops through an interference with blood supply to the central nervous system and may be associated with oxidative stress i.e. an imbalance between ROS production and metabolism [17]. Although low level generation of ROS is critical for neuronal homeostasis, their excess generation, particularly by NAD $(\mathrm{P}) \mathrm{H}$ oxidase, is implicated in vascular injuries and intracranial haemorrhage [18, 19]. Increases in certain NAD $(\mathrm{P}) \mathrm{H}$ oxidase subunit expressions have been shown in STZ-induced diabetic rats with transient middle cerebral artery occlusion, an animal model of human ischaemic stroke and correlated with infarct sizes, more severe neurological deficits and brain oedema volume [20]. Significant increases in NAD(P)H oxidase enzyme activity and p22-phox protein expression, an integral $\mathrm{NAD}(\mathrm{P}) \mathrm{H}$ subunit, have also been found in this study in HBMEC cultured under hyperglycaemic conditions. Given that these increases were suppressed by normalisation of glucose levels, collectively these data shed some light on the mechanistic causes of HG-mediated increases of oxidative stress and subsequent endothelial dysfunction.

As deficiencies in MnSOD and/or CuZnSOD activities may also contribute to enhanced oxidative status, the expression and activities of these enzymes were explored in HBMEC exposed to HG which revealed no changes or significant increases in cases of MnSOD and CuZnSOD, respectively. Since HG significantly increases the BBB permeability, these data imply that the increase in CuZnSOD activity ( 2-fold) which makes up $\sim 80 \%$ of total SOD activity [21] is not sufficient to quench the excessive ROS generated under hyperglycaemic conditions. In support of this hypothesis, the current study has shown that the concurrent 
employment of HG with one of the two structurally different SOD mimetics i.e. MnTBAP or Mn(III)TMPyP attenuates BBB hyperpermeability. Higher rates of increases in CuZnSOD has previously been shown to diminish HG-enhanced brain oedema and Evans blue leakage in heterozygous SOD1 Tg rats carrying human SOD1 genes to achieve 4- to 6-fold increase in $\mathrm{CuZnSOD} \mathrm{[22].} \mathrm{It} \mathrm{is} \mathrm{of} \mathrm{note} \mathrm{that} \mathrm{while} \mathrm{normalisation} \mathrm{of} \mathrm{glucose} \mathrm{levels} \mathrm{effectively} \mathrm{suppressed}$ CuZnSOD activity, it had no effect on its protein expression. A compensatory response to increase subdued enzyme activity by de novo protein synthesis in order to neutralise still considerably high quantities of $\mathrm{O}_{2}{ }^{--}$during normoglycaemic phase following a hyperglycaemic insult may, in part, account for this phenomenon.

Once dismutated $\mathrm{O}_{2}{ }^{--}$forms $\mathrm{H}_{2} \mathrm{O}_{2}$ which requires further breakdown to $\mathrm{H}_{2} \mathrm{O}$ by antioxidant enzymes catalase and GPx. This study has shown significant increases in catalase activity and protein expression in $\mathrm{HBMEC}$ exposed to $\mathrm{HG}$ which were reversed by glucose normalisation and consequent removal of HG-mediated oxidative insult. Similar to catalase, a slight but significant increase was also observed in GPx protein expression in cells cultured under hyperglycaemic conditions. However, the increase in protein levels was not translated into activity perhaps as a result of previously reported instability of the GPx complex over time in diabetic rat tissues including brain [23].

NO, another ROS, has also been implicated in the BBB hyperpermeability in cerebral pathologies such as middle cerebral artery occlusion [12]. It is mainly generated by endothelial NO synthase (eNOS) whose activity and expression under diabetic conditions remain controversial in that both enhanced and diminished eNOS expressions have been reported in diabetic patients, animal models and also in cells cultured with high concentrations of glucose $[24,25]$. However, similar to a previous study showing substantial elevations in STZ-induced diabetic rat hippocampus, cortex, cerebellum, brain stem and spinal cord [26], the current study has also revealed considerable increases in both eNOS 
enzyme activity and nitrite levels in HBMEC subjected to HG which were attenuated by glucose normalisation. Although these findings may suggest a possibility that, in contrast to peripheral organs eNOS may be regulated in a similar manner by HG in different parts of the central nervous system, this hypothesis requires further detailed investigations.

Endothelial dysfunction is in part regulated by the expression of adhesion molecules such as ICAM-1 and VCAM-1 on the surface of ECs. Results from this study demonstrate that VCAM-1 but not ICAM-1 concentrations are increased under HG conditions which are dramatically decreased by glucose normalisation. Similar to our study, Haubner et al have also reported a glucose-specific increase in the basal expression of membrane-bound VCAM1 but not ICAM-1 and E-Selectin [27]. Conversely, a recent in vivo study has shown that the numbers of ICAM-1 positively stained microvessels in the cortex were markedly increased at 3 days of reperfusion in diabetic but not in non-diabetic rats, suggesting that inflammatory responses mediate HG-aggravated brain damage, induced by ischaemia and reperfusion [28]. Although the reasons behind the differences between in vitro and in vivo studies require exploration, development of intracerebral diabetic ketoacidosis may in part account for this dichotomy. Indeed, an in vitro study with increasing concentrations of glucose along with acetoacetate and $\beta$-hydroxybutyrate led to increased expression of ICAM-1 and perturbed membrane function [29].

It is well-documented that the integrity of the BMEC layer is critical for BBB function as a whole and pathological conditions including HG may compromise this tight continuum through facilitating the formation of openings in transcellular or paracellular pathways. Hence, the last phase of the current study examined the alterations in BBB permeability under hyperglycaemic versus normoglycaemic conditions via TEER measurements. As static monolayer and non-contact co-culture models did not produce significant variations in TEER, contact co-cultures have been used throughout the study. The current study has shown that 
HG exerted a significant decrease in TEER, a reliable indicator of the BBB integrity which was blocked by a series of antioxidants with unique mechanisms of action thereby proving oxidative stress as a key player in the pathogenesis of this defect. The antioxidant compounds used in the study were vitamin C (an antioxidant vitamin), apocynin (a specific inhibitor of NAD(P)H oxidase), MPG ( $\mathrm{O}_{2}^{-{ }^{-}}$scavenger), MnTBAP and Mn(III)TMPyP (SOD mimetics), catalase (an antioxidant enzyme) and Bis-I and SB203580 (protein kinase C and p38MAPK inhibitors, respectively). These findings were in agreement with previous studies showing that SOD and catalase administrations reduce the tracer permeation to the cerebral ischemic brain in rats [30-32].

The findings with the PKC inhibitor were, to a large degree, expected considering undisputed involvement of PKC activation in hyperglycaemia-mediated peripheral vasculopathies, ROS overproduction and cerebral endothelial dysfunction [7, 8, 33]. Protein kinase C may utilise two mechanisms to impair NO-mediated vasodilatation. Firstly, it may directly modify eNOS post-translationally via its dephosphorylation at Ser1177 and phosphorylation at Thr495 which together result in decreased NO production [34]. Secondly, protein kinase $\mathrm{C}$ may impair eNOS function indirectly through effects on $\mathrm{NAD}(\mathrm{P}) \mathrm{H}$ oxidase, the most potent generator of $\mathrm{O}_{2}{ }^{--}$that rapidly scavenges NO. Similar to PKC, inhibition of p38MAPK has also been shown as neuroprotective in cerebral ischaemia $[35,36]$. The p38 pathway may mediate neuronal damage by counteracting ERK signalling in models of apoptosis and free radical damage in vitro [38], by controlling the release of granules in neutrophils in the process of inflammation [39] or by induction of cytokines [40]. However, contradictory reports with p38MAPK inhibitor also exist in that rats given SB203580 prior to transient middle cerebral artery occlusion have been shown to develop widespread cerebral lesions and vascular leakage [37]. 
Research encompassing the PI3K inhibitor, LY29402 is very limited. However, Gottfried et al reported loss of polygonal morphology with varying degrees of stellation in rat hippocampal astrocytes treated with LY29402 [41]. This may have significance as intact brain astrocytic processes encounter and bind to collagen IV and laminin in the basement membrane of endothelial cells that surround cerebral capillaries and form the BBB [42].

Concurrence between previously published data and our findings suggest that the potential therapies for hyperglycaemia in acute stroke patients should include treatment regimens that establish and maintain normoglycaemia. In support of this notion, a recent retrospective study of AIS patients showed that patients with an admission glucose level of $>130 \mathrm{mg} / \mathrm{dl}$ had increased mortality compared to those whose glucose levels were normalised within $48 \mathrm{hr}$ [43]. In this context, another study has revealed that intensive intravenous insulin therapy to maintain NG reduce the risk of organ failure and death in surgical intensive care patients [44].

In conclusion the current study has shown that HBMEC incubated with HG possess enhanced $\mathrm{NAD}(\mathrm{P}) \mathrm{H}$ oxidase enzyme activity and expression compared to cells grown under NG and that the reversal of HG to NG significantly suppress these increases. Secondly the increased production of free radicals by $\mathrm{NAD}(\mathrm{P}) \mathrm{H}$ oxidase through activation of different signal transduction pathways appear to compromise the integrity of the BBB under diabetic conditions. Effective reversal of high glucose levels exerts beneficial effects on the preservation of the BBB unity and may therefore be therapeutically beneficial in diabetic stroke patients.

\section{Acknowledgements}

This study was supported by grants to Dr Bayraktutan from the Nottingham University Hospital Special Trustees and the University of Nottingham New Researchers Fund. 


\section{References}

1. Bayraktutan U. Free radicals, diabetes and endothelial dysfunction. Diabetes Obes Metab 2002; 4: 224-238.

2. Brownlee M. Biochemistry and molecular cell biology of diabetic complications. Nature 2001; 414: 813-820.

3. Abbott N. Astrocyte-endothelial interactions and blood-brain barrier permeability. J Anat 2002; 200: 527.

4. Baird TA, Parsons MW, Barber PA et al. The influence of diabetes mellitus and hyperglycaemia on stroke incidence and outcome. J Clin Neurosci 2002; 9: 618-626.

5. Hacke W, Schwab S, Horn M et al. 'Malignant' middle cerebral artery territory infarction: clinical course and prognostic signs. Arch Neurol 1996; 53: 309-315.

6. Kiers L, Davis SM, Larkins R et al. Stroke topography and outcome in relation to hyperglycaemia and diabetes. J Neurol Neurosurg Psychiatry 1992; 55: 263-270.

7. Bayraktutan U, Yang ZK, Shah AM. Selective dysregulation of nitric oxide synthase type 3 in cardiac myocytes but not coronary microvascular endothelial cells of spontaneously hypertensive rat. Cardiovasc Res 1998; 38: 719-726.

8. Cai S, Khoo J, Channon KM. Augmented BH4 by gene transfer restores nitric oxide synthase function in hyperglycemic human endothelial cells. Cardiovasc Res 2005; 65: $823-831$.

9. Capes SE, Hunt D, Malmberg K, Pathak P, Gerstein HC. Stress hyperglycemia and prognosis of stroke in nondiabetic and diabetic patients: a systematic overview. Stroke 2001; 32: 2426-2432.

10. Ago T, Kitazono T, Kuroda J et al. NAD(P)H oxidases in rat basilar arterial endothelial cells. Stroke 2005; 36: 1040-1046. 
11. Ceriello A, dello Russo P, Amstad P, Cerutti P. High glucose induces antioxidant enzymes in human endothelial cells in culture. Evidence linking hyperglycemia and oxidative stress. Diabetes 1996; 45: 471-477.

12. Traystman RJ, Moore LE, Helfaer MA et al. Nitro-L-arginine analogues. Dose- and time-related nitric oxide synthase inhibition in brain. Stroke 1995; 26: 864-869.

13. Morikawa E, Moskowitz MA, Huang Z et al. L-arginine infusion promotes nitric oxide-dependent vasodilation, increases regional cerebral blood flow, and reduces infarction volume in the rat. Stroke 1994; 25: 429-435.

14. Grau AJ, Weimar C, Buggle F et al. Risk factors, outcome, and treatment in subtypes of ischemic stroke: the German stroke data bank. Stroke 2001; 32: 2559-2566.

15. Duckrow RB, Beard DC, Brennan RW. Regional cerebral blood flow decreases during hyperglycemia. Ann Neurol 1985; 17: 267-272.

16. Bell DS. Stroke in the diabetic patient. Diabetes Care 1994; 17: 213-219.

17. Hou ST, MacManus JP. Molecular mechanisms of cerebral ischemia-induced neuronal death. Int Rev Cytol 2002; 221: 93-148.

18. Crack PJ, Taylor JM. Reactive oxygen species and the modulation of stroke. Free Radic Biol Med 2005; 38: 1433-1444.

19. Guzik TJ, West NE, Black E et al. Vascular superoxide production by NAD(P)H oxidase: association with endothelial dysfunction and clinical risk factors. Circ Res 2000; 86: E85-90.

20. Kusaka I, Kusaka G, Zhou C et al. Role of AT1 receptors and NAD(P)H oxidase in diabetes-aggravated ischemic brain injury. Am J Physiol Heart Circ Physiol 2004; 286: H2442-2451.

21. Didion SP, Ryan MJ, Didion LA et al. Increased superoxide and vascular dysfunction in CuZnSOD-deficient mice. Circ Res 2002; 91: 938-944. 
22. Kamada H, Yu F, Nito C, Chan PH. Influence of hyperglycemia on oxidative stress and matrix metalloproteinase-9 activation after focal cerebral ischemia/reperfusion in rats: relation to blood-brain barrier dysfunction. Stroke 2007; 38: 1044-1049.

23. Ulusu NN, Sahilli M, Avci A et al. Pentose phosphate pathway, glutathione-dependent enzymes and antioxidant defense during oxidative stress in diabetic rodent brain and peripheral organs: effects of stobadine and vitamin E. Neurochem Res 2003; 28: 815823.

24. Cosentino F, Hishikawa K, Katusic ZS, Luscher TF. High glucose increases nitric oxide synthase expression and superoxide anion generation in human aortic endothelial cells. Circulation 1997; 96: 25-28.

25. Chakravarthy U, Hayes RG, Stitt AW, McAuley E, Archer DB. Constitutive nitric oxide synthase expression in retinal vascular endothelial cells is suppressed by high glucose and advanced glycation end products. Diabetes 1998; 47: 945-952.

26. Ates O, Yucel N, Cayli SR et al. Neuroprotective effect of etomidate in the central nervous system of streptozotocin-induced diabetic rats. Neurochem Res 2006; 31: 777-783.

27. Haubner F, Lehle K, Munzel D et al. Hyperglycemia increases the levels of vascular cellular adhesion molecule- 1 and monocyte-chemoattractant-protein- 1 in the diabetic endothelial cell. Biochem Biophys Res Commun 2007; 360: 560-565.

28. Ding Y, Li J, Rafols JA, Phillis JW, Diaz FG. Prereperfusion saline infusion into ischemic territory reduces inflammatory injury after transient middle cerebral artery occlusion in rats. Stroke 2002; 33: 2492-2498.

29. Hoffman WH, Cheng C, Passmore GG, Carroll JE, Hess D. Acetoacetate increases expression of intercellular adhesion molecule-1 (ICAM-1) in human brain microvascular endothelial cells. Neurosci Lett 2002; 334: 71-74. 
30. Armstead WM, Mirro R, Thelin OP et al. Polyethylene glycol superoxide dismutase and catalase attenuate increased blood-brain barrier permeability after ischemia in piglets. Stroke 1992; 23: 755-762.

31. Liu ZF, Fang YZ. Observations on the activity and immunologic properties of CuZnSOD of whole blood in cancer patients. Zhonghua Yi Xue Za Zhi 1989; 69: 212-213.

32. Nelson CW, Wei EP, Povlishock JT, Kontos HA, Moskowitz MA. Oxygen radicals in cerebral ischemia. Am J Physiol 1992; 263: H1356-1362.

33. Mayhan WG, Didion SP. Activation of protein kinase $\mathrm{C}$ does not participate in disruption of the blood-brain barrier to albumin during acute hypertension. Brain Res 1995; 696: 106-112.

34. Fleming N, Mellow L. Distribution and translocation of isoforms of protein kinase C in rat submandibular acinar cells. Life Sci 1995; 57: 2003-2010.

35. Barone FC, Irving EA, Ray AM et al. Inhibition of p38 mitogen-activated protein kinase provides neuroprotection in cerebral focal ischemia. Med Res Rev 2001; 21: 129-145.

36. Sugino T, Nozaki K, Hashimoto N. Activation of mitogen-activated protein kinases in gerbil hippocampus with ischemic tolerance induced by 3-nitropropionic acid. Neurosci Lett 2000; 278: 101-104.

37. Lennmyr F, Ericsson A, Gerwins P, Ahlstrom H, Terent A. Increased brain injury and vascular leakage after pretreatment with p38-inhibitor SB203580 in transient ischemia. Acta Neurol Scand 2003; 108: 339-345.

38. Xia Z, Dickens M, Raingeaud J, Davis RJ, Greenberg ME. Opposing effects of ERK and JNK-p38 MAP kinases on apoptosis. Science 1995; 270: 1326-1331. 
39. Mocsai A, Jakus Z, Vantus T et al. Kinase pathways in chemoattractant-induced degranulation of neutrophils: the role of p38 mitogen-activated protein kinase activated by Src family kinases. J Immunol 2000; 164: 4321-4331.

40. Lee JC, Laydon JT, McDonnell PC et al. A protein kinase involved in the regulation of inflammatory cytokine biosynthesis. Nature 1994; 372: 739-746.

41. Gottfried C, Cechin SR, Gonzalez MA, Vaccaro TS, Rodnight R. The influence of the extracellular matrix on the morphology and intracellular $\mathrm{pH}$ of cultured astrocytes exposed to media lacking bicarbonate. Neuroscience 2003; 121: 553-562.

42. Tilling T, Engelbertz C, Decker S et al. Expression and adhesive properties of basement membrane proteins in cerebral capillary endothelial cell cultures. Cell Tissue Res 2002; 310: 19-29.

43. Gentile NT, Seftchick MW, Huynh T, Kruus LK, Gaughan J. Decreased mortality by normalizing blood glucose after acute ischemic stroke. Acad Emerg Med 2006; 13: 174-180.

44. van den Berghe G, Wouters P, Weekers F et al. Intensive insulin therapy in the critically ill patients. N Engl J Med 2001; 345: 1359-1367. 


\section{Figure legends}

Fig. 1 (A) Representative Western blots of pro-oxidant (p22-phox) and anti-oxidant proteins in human brain microvascular endothelial cells (HBMEC) cultured under hyperosmolality (lane 1; 25mM D-mannitol), normoglycaemia (lane 2; 25mM D-Glucose), hyperglycaemia (lane 3; $25 \mathrm{mM}$ D-Glucose) and hyperglycaemia followed by normoglycaemia (lane 4) for a period of 5 days. (B) Histograms showing fold-differences in anti-oxidant and pro-oxidant protein expressions in $\mathrm{HBMEC}$ exposed to $\mathrm{HG}$ versus $\mathrm{NG}$ or $\mathrm{HG} / \mathrm{NG}$. Data are expressed as mean \pm SEM from 3 different experiments. ${ }^{*}$ p $<0.05$ HG vs NG. ${ }^{*}$ p $<0.05$ HG vs HG/NG.

Fig. 2 The effects of hyperglycaemia (25mM D-Glucose) and glucose normalisation on human brain microvascular endothelial cell expression intercellular adhesion molecule-1 (ICAM-1; A) and vascular cell adhesion molecule-1 (VCAM-1, B) following 5 days of treatment. Data are expressed as mean \pm SEM from 5 different experiments. ${ }^{*} \mathrm{p}<0.05$ compared to NG (5 mM D-Glucose). ${ }^{\#} \mathrm{p}<0.05$ compared to HG (25mM D-Glucose).

Fig. 3 (A) Schematic diagram of the in vitro models of the BBB and corresponding readings of transendothelial electrical resistance readings (TEER; B). (1) Control, (2) Human brain endothelial cell (HBMEC), (3) Human astrocyte (HA) and HBMEC non-contact (4) HA and HBMEC contact cultures. Data are expressed as mean \pm SEM from 5 different experiments. ${ }^{*} \mathrm{p}<0.05$ compared to non-contact model.

Fig. 4 Effects of hyperosmolality (25mM D-mannitol), normoglycaemia (5.5.mM DGlucose), hyperglycaemia (25mM D-Glucose) and hyperglycaemia followed by normoglycaemia on BBB permeability. Data are expressed as mean \pm SEM from 5 different experiments. ${ }^{*} \mathrm{p}<0.05$ compared to NG (5 mM D-Glucose). ${ }^{*} \mathrm{p}<0.05$ compared to HG $(25 \mathrm{mM}$ D-Glucose).

Fig. 5 Effects of antioxidants, apocynin $(500 \mu \mathrm{M})$, vitamin $\mathrm{C}(0.5 \mu \mathrm{M}), \mathrm{MPG}(0.5 \mu \mathrm{M})$, and superoxide dismutase mimetic MnTBAP (50 $\mu \mathrm{M})$ and $\mathrm{Mn}(\mathrm{III}) \mathrm{TMPyP}(10 \mu \mathrm{M})$ and catalase 
(25 and150 U/mL) on transendothelial electrical resistance (TEER) readings. Data are expressed as mean \pm SEM from 5 different experiments. ${ }^{*} \mathrm{p}<0.05$ compared to NG (5 mM DGlucose). ${ }^{\#}$ p $<0.05$ compared to HG (25mM D-Glucose).

Fig. 6 Effects of protein kinase C (Bisindolyamide I, $5 \mu \mathrm{M})$, p38MAPK (SB203580, $10 \mu \mathrm{M})$ and PI-3 kinase (LY29402, $50 \mu \mathrm{M}$ ) inhibitors on transendothelial electrical resistance (TEER). Data are expressed as mean \pm SEM from 6 different experiments. ${ }^{*} \mathrm{p}<0.05$ compared to $\mathrm{NG}$ (5.5mM D-Glucose). ${ }^{\#} \mathrm{p}<0.05$ compared to HG (25mM D-Glucose). 
Figure 1.

A.

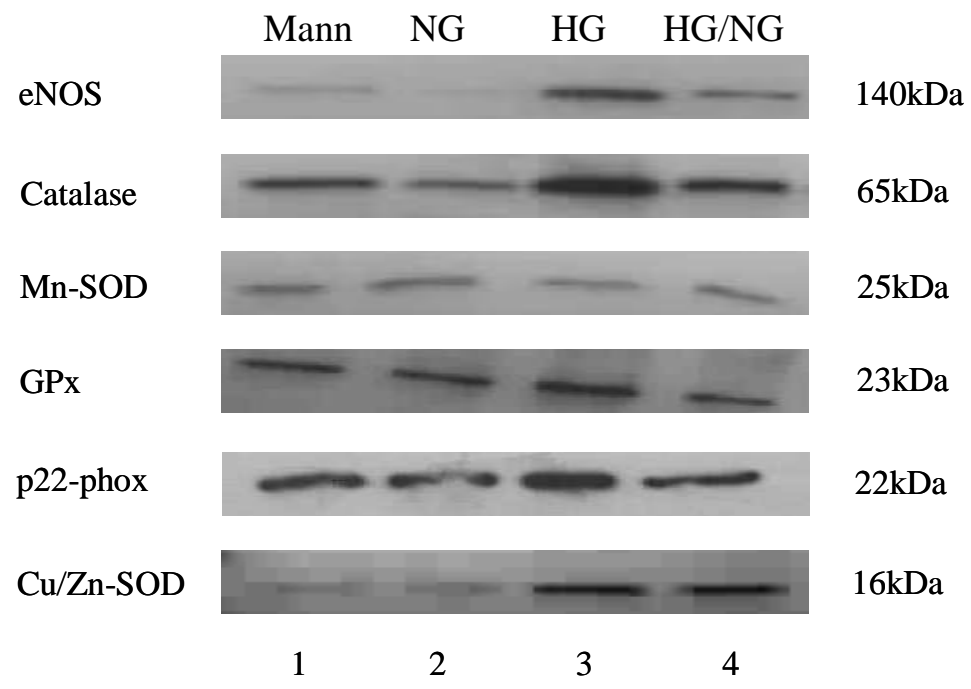

B.

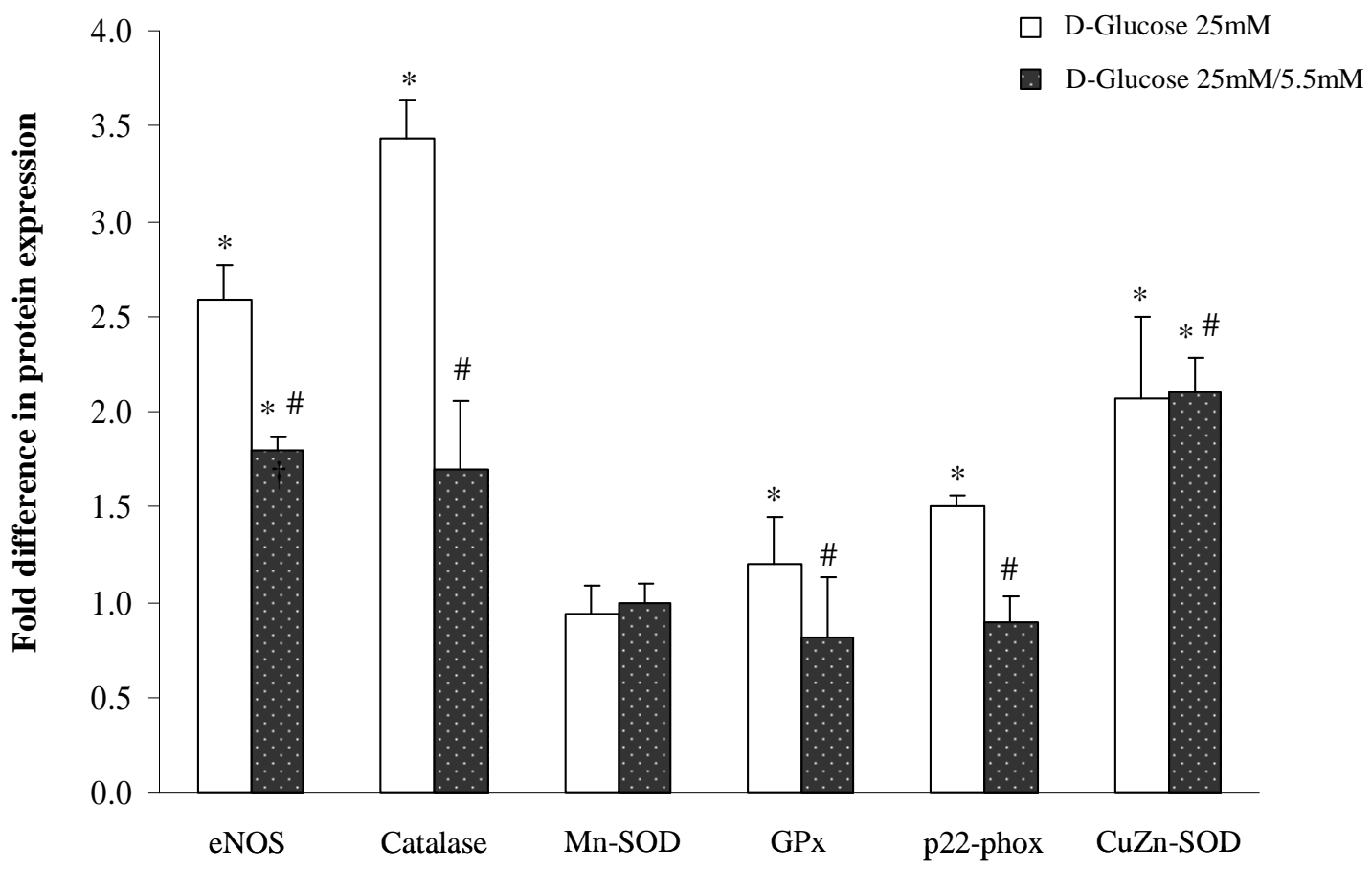


Figure 2.

A.

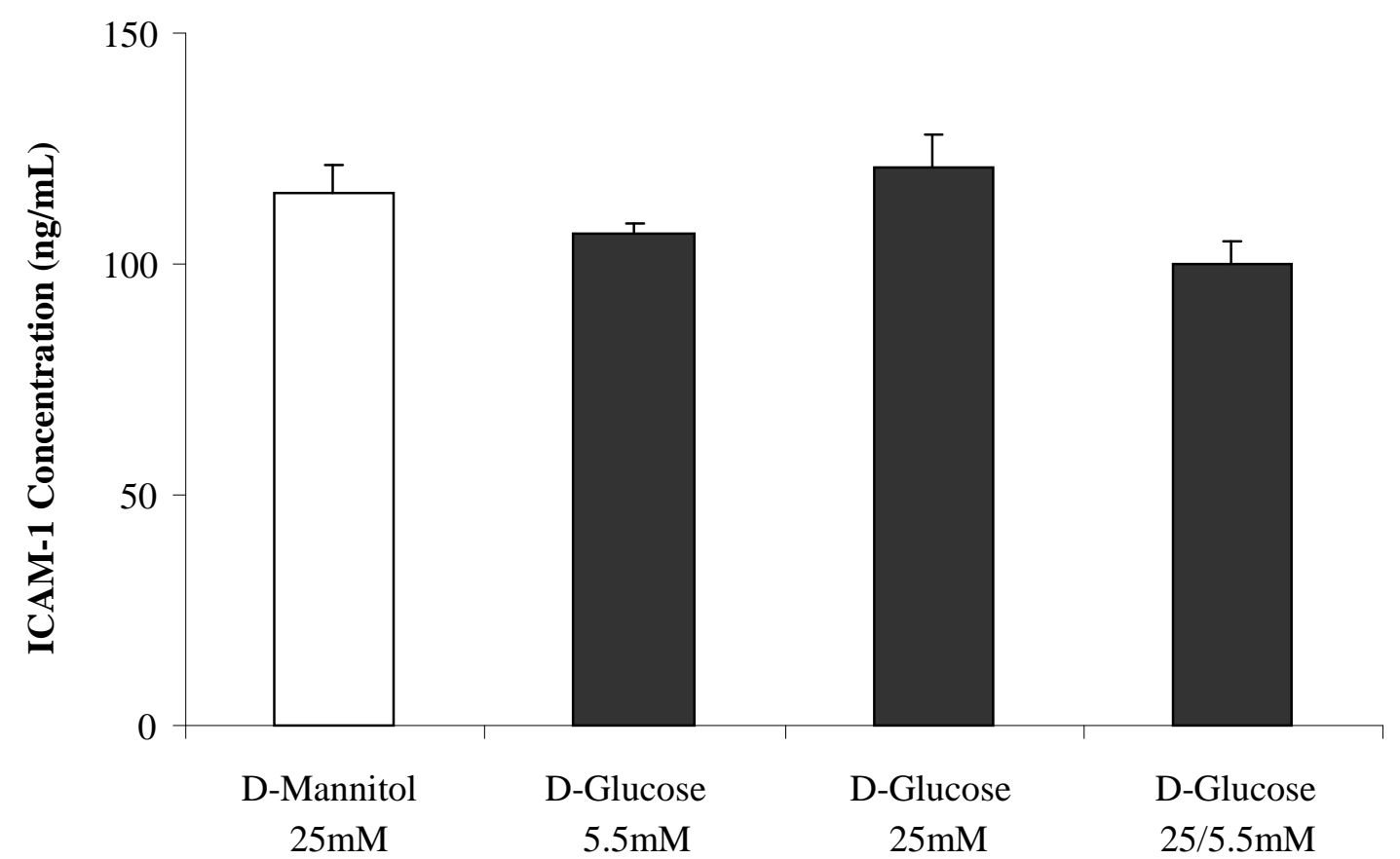

B.

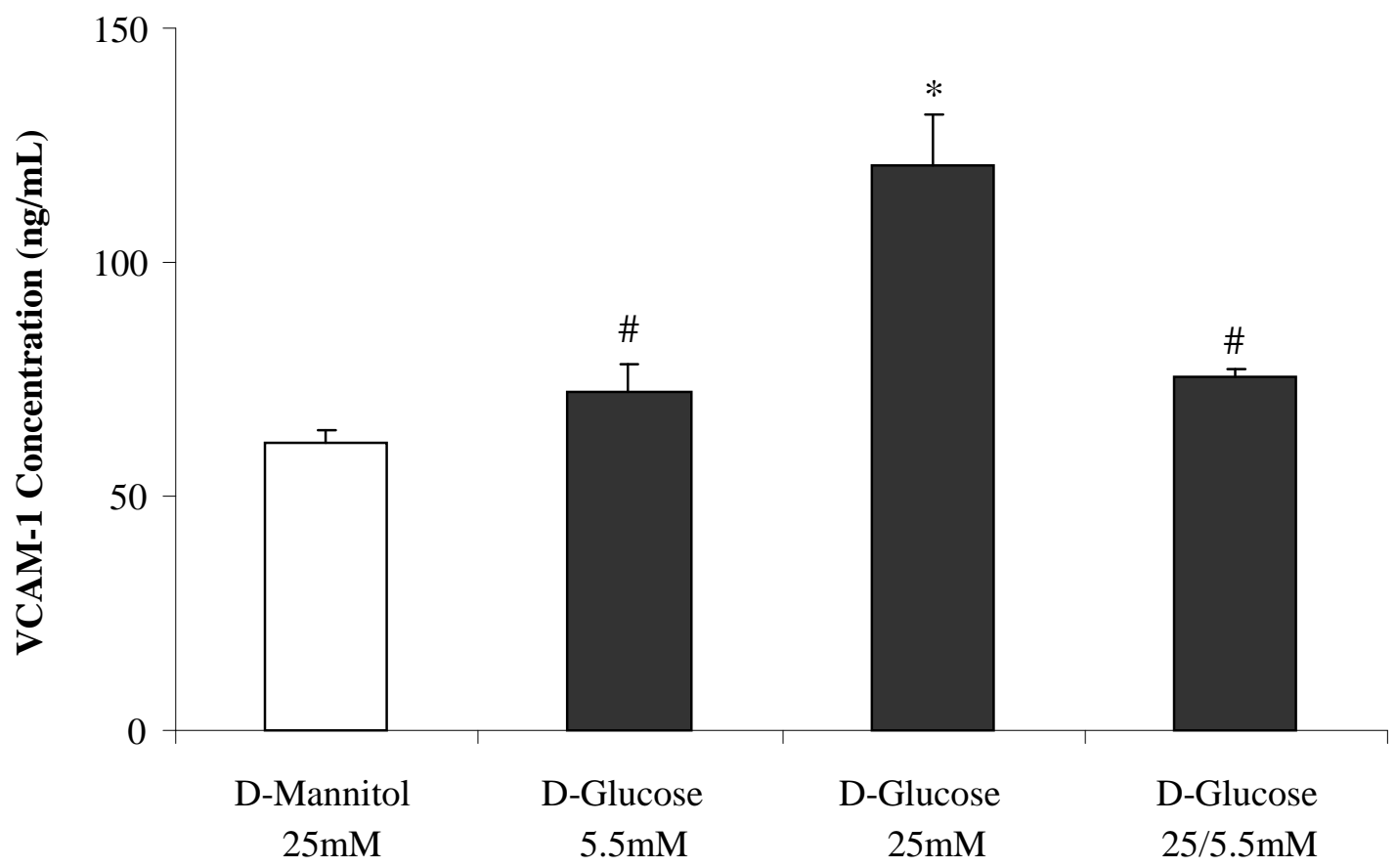


Figure 3.

A.

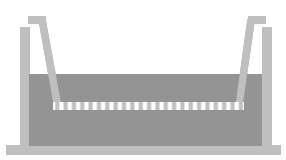

Control

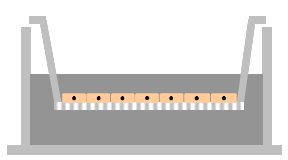

Monolayer

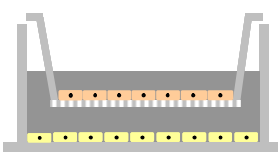

Non-contact

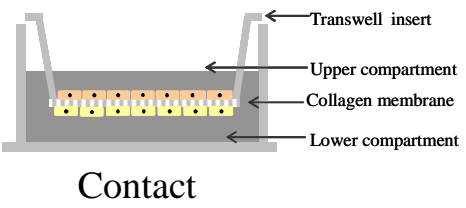

Contact

B.

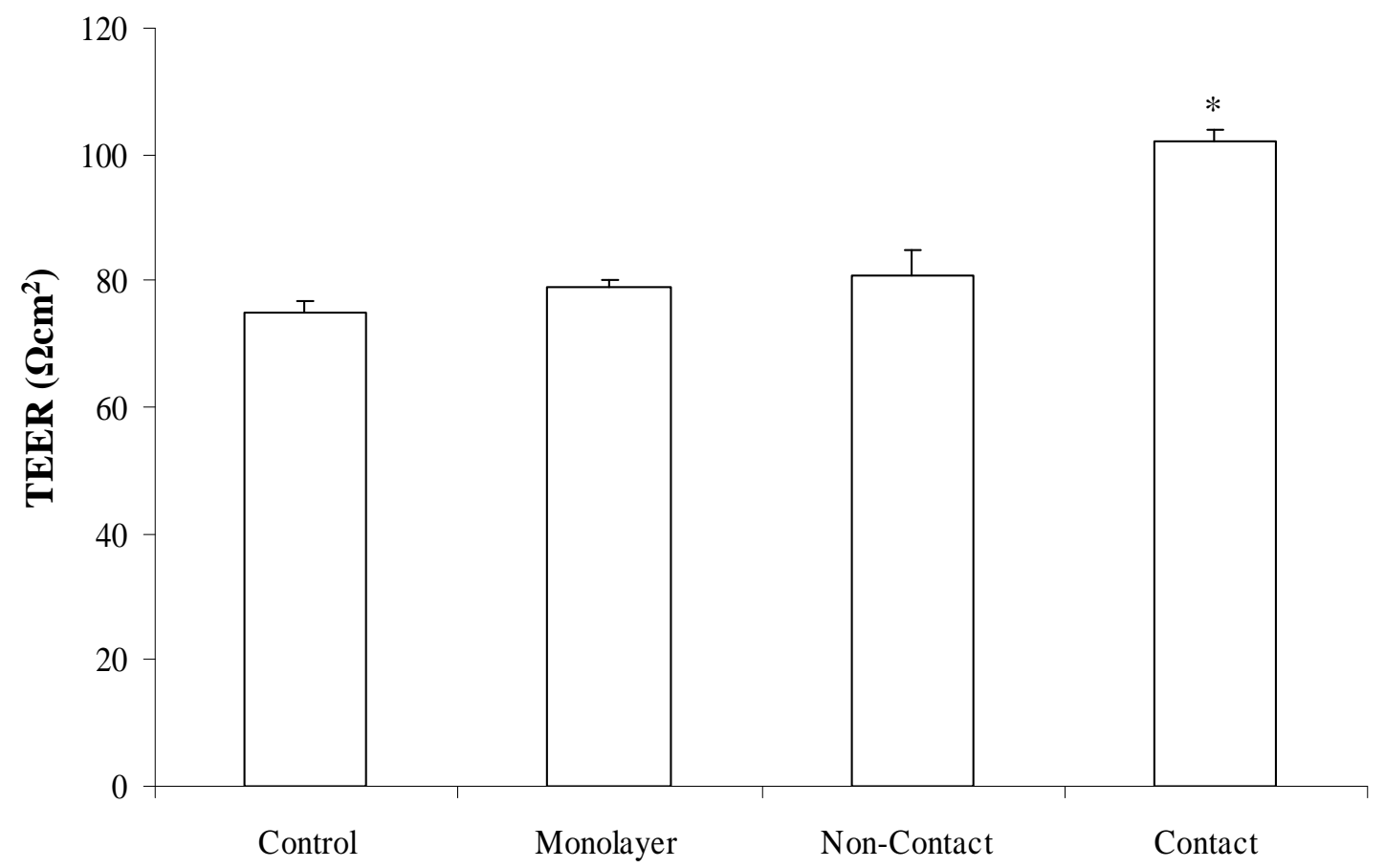


Figure 4.

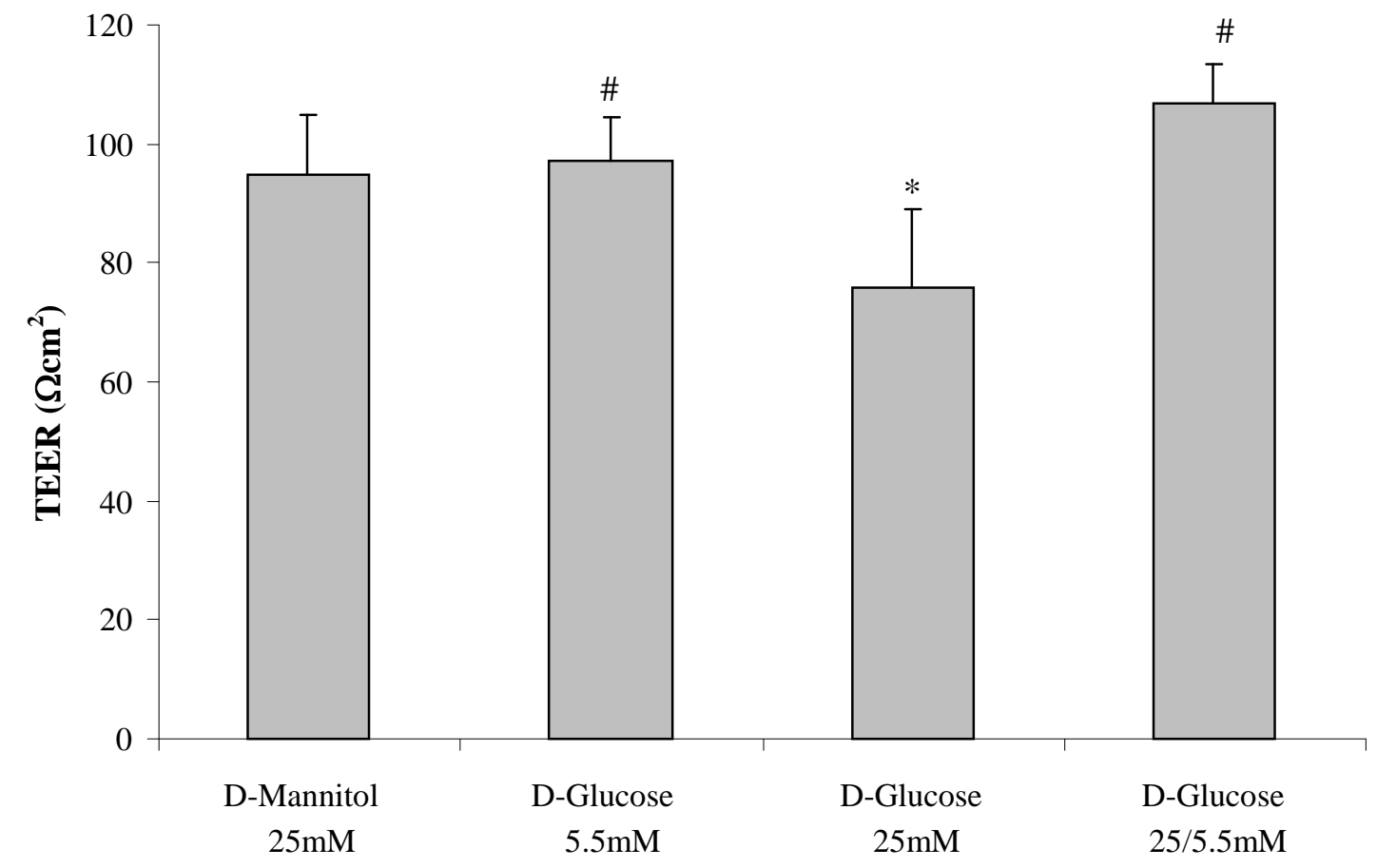


Figure 5.

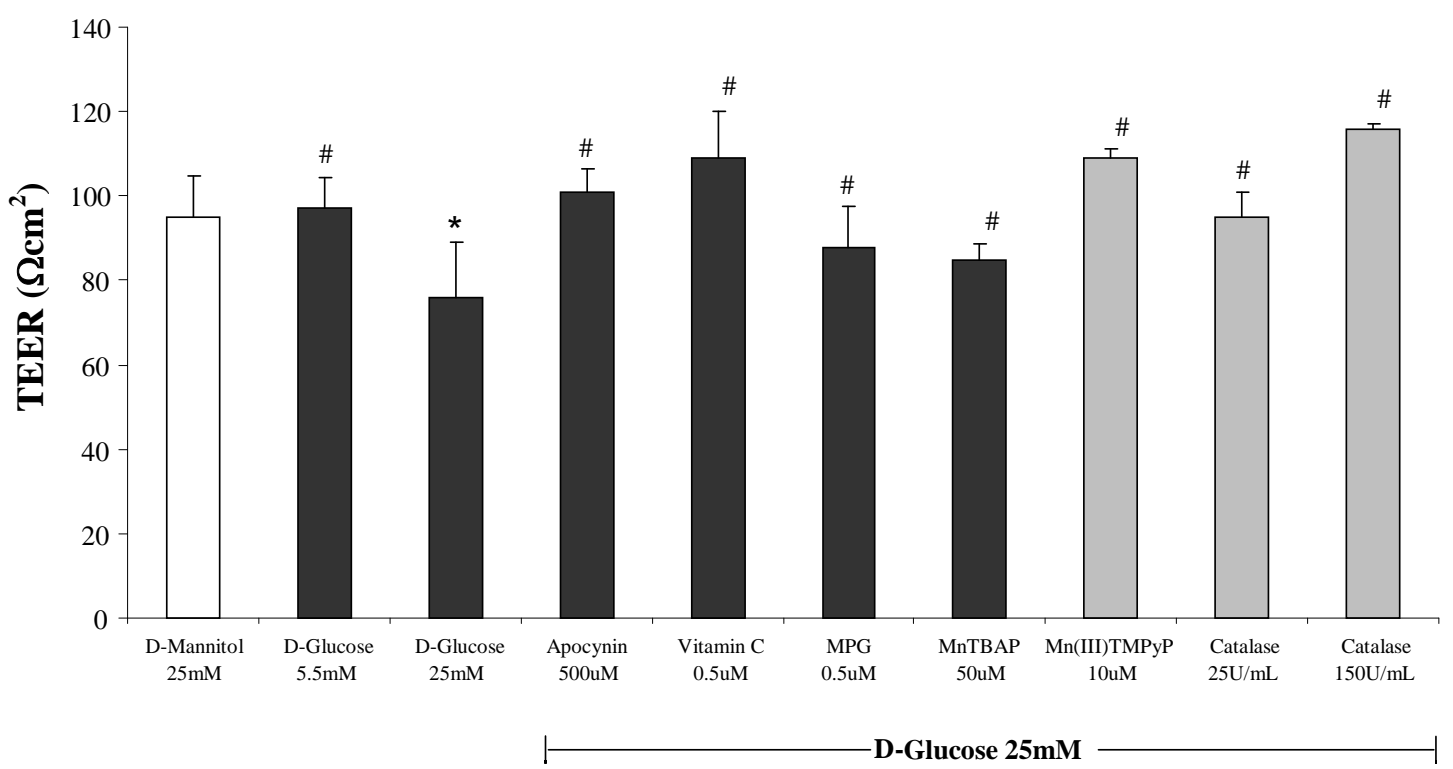


Figure 6.

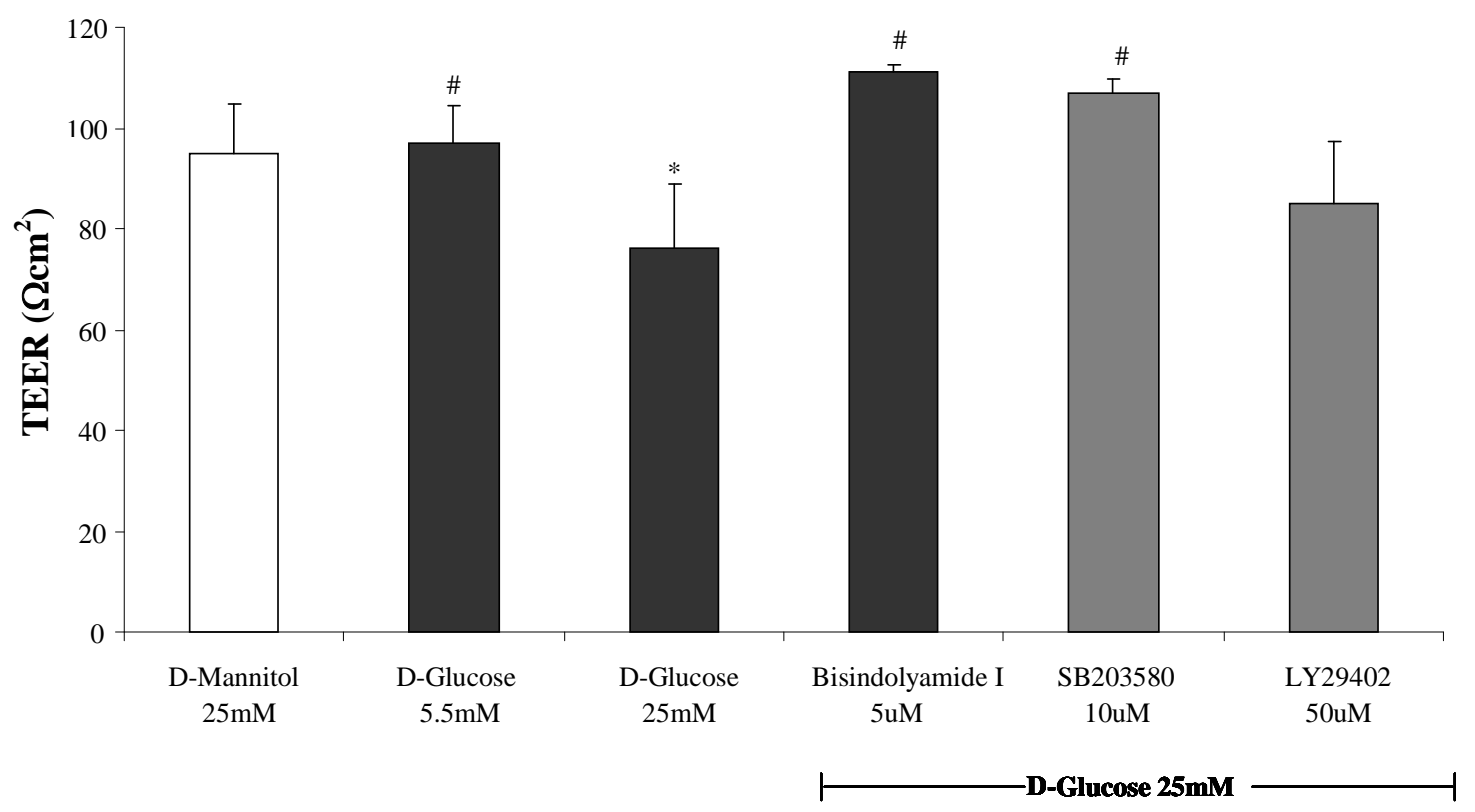


Table 1. The effects of hyperglycaemia and glucose normalisation on prooxidant NAD(P)H oxidase and antioxidant enzyme activities.

\begin{tabular}{|c|c|c|c|c|c|c|c|}
\hline Treatment Group & $\begin{array}{l}\mathrm{NAD}(\mathrm{P}) \mathrm{H} \text { Oxidase } \\
\text { (pmole } \mathrm{O}_{2}-\text { mg protein) }\end{array}$ & $\begin{array}{c}\text { eNOS } \\
\text { (pmole L-citrulline/mg } \\
\text { protein/min) }\end{array}$ & $\begin{array}{c}\text { CuZnSOD } \\
\text { (mU/mg protein) }\end{array}$ & $\begin{array}{c}\text { MnSOD } \\
\text { (mU/mg protein) }\end{array}$ & $\begin{array}{c}\text { GPx } \\
(\mathrm{nmol} / \mathrm{min} / \mathrm{mL})\end{array}$ & $\begin{array}{c}\text { Catalase } \\
(\mathrm{nmol} / \mathrm{min} / \mathrm{mL})\end{array}$ & $\begin{array}{l}\text { Nitrite } \\
(\mu \mathrm{M})\end{array}$ \\
\hline D-Mannitol 25mM & $0.042 \pm 0.007$ & $13415.9 \pm 886.5$ & $278 \pm 46$ & $12 \pm 1$ & $64.09 \pm 6.61$ & $22.13 \pm 1.97$ & $10.44 \pm 0.67$ \\
\hline D-Glucose $5.5 \mathrm{mM}$ & $0.045 \pm 0.007^{\dagger}$ & $12952.8 \pm 924.4$ & $290 \pm 37^{\dagger}$ & $24 \pm 6$ & $53.40 \pm 4.88$ & $21.94 \pm 0.59$ & $6.73 \pm 0.86$ \\
\hline D-Glucose $25 \mathrm{mM}$ & $0.100 \pm 0.006^{*}$ & $17361.4 \pm 1414.9^{*}$ & $428 \pm 39^{*}$ & $22 \pm 3$ & $58.92 \pm 6.40$ & $27.00 \pm 1.56^{*}$ & $13.44 \pm 0.24^{*}$ \\
\hline D-Glucose $25 / 5.5 \mathrm{mM}$ & $0.067 \pm 0.005^{* \dagger}$ & $15720.6 \pm 277.9^{* \dagger}$ & $209 \pm 26^{* \dagger}$ & $7 \pm 4 * \dagger$ & $21.83 \pm 5.40^{* \dagger}$ & $10.36 \pm 1.22^{* \dagger}$ & $10.77 \pm 0.37^{* \dagger}$ \\
\hline
\end{tabular}

The levels of enzyme activities were measured in human brain microvascular endothelial cells (HBMEC) cultured with a hyperosmolality control (D-Mannitol 25mM),

normal glucose (D-Glucose 5.5mM), high glucose (D-Glucose $25 \mathrm{mM})$ and high glucose followed by normal glucose for a period of 5 days. Data are expressed as mean \pm

SEM from 5 different experiments. ${ }^{*} \mathrm{p}<0.05$ compared to $\mathrm{NG}$ (D-Glucose $\left.5.5 \mathrm{mM}\right) .{ }^{\dagger} \mathrm{p}<0.05$ compared to HG (D-Glucose $\left.25 \mathrm{mM}\right)$. 
Table 2. Effects of hyperosmolality, normoglycaemia, hyperglycaemia and hyperglycaemia followed by normoglycaemia on HBMEC and HA viability.

\begin{tabular}{ccc}
\hline Treatment Group & HBMEC & HA \\
\hline D-Mannitol 25mM & $86 \pm 4$ & $91 \pm 4$ \\
D-Glucose 5.5mM & $93 \pm 5$ & $90 \pm 7$ \\
D-Glucose 25mM & $89 \pm 2$ & $88 \pm 5$ \\
D-Glucose 25/5.5mM & $90 \pm 7$ & $87 \pm 6$ \\
\hline
\end{tabular}

Percentage viable cells were determined by trypan blue exclusion experiments and counting 100 cells. Data are expressed as mean \pm SEM from 3 different experiments. 